\title{
e-Phaïstos
}

e-Phaïstos

Revue d'histoire des techniques / Journal of the history

of technology

VIII-1 | 2020

Écomusée : une expansion internationale

\section{Écomusées en Europe de l'Est : histoire et avenir}

Ecomuseums in Eastern Europe : History and Future

\section{Mihaela Nichifor}

\section{OpenEdition}

Journals

Édition électronique

URL : http://journals.openedition.org/ephaistos/7472

DOI : $10.4000 /$ ephaistos.7472

ISSN : 2552-0741

Éditeur

IHMC - Institut d'histoire moderne et contemporaine (UMR 8066)

Référence électronique

Mihaela Nichifor, «Écomusées en Europe de l'Est : histoire et avenir », e-Phaïstos [En ligne], VIII-1 |

2020, mis en ligne le 29 avril 2020, consulté le 06 mars 2021. URL : http://journals.openedition.org/ ephaistos/7472 ; DOI : https://doi.org/10.4000/ephaistos.7472

Ce document a été généré automatiquement le 6 mars 2021.

Tous droits réservés 


\title{
Écomusées en Europe de l'Est : histoire et avenir
}

\author{
Ecomuseums in Eastern Europe : History and Future
}

Mihaela Nichifor

«[... Car l'écomusée est devenu trop souvent une
mode, un moyen de donner une image plus
moderne au vieux musée d'autrefois, sans pour
autant modifier le fond des choses, définir
l'objectif de l'initiative, approfondir la
signification de ses actions ${ }^{1}$ ", Hugues de Varine.

Dès leur apparition, il y a une cinquantaine d'années (1971), les écomusées sont devenus un must-have dans le monde. D'après Hugues de Varine, ils sont une sorte de nouvelle mode parmi les types de musées: l'image de la modernisation des musées traditionnels qui gardent, quand même, le sens des choses, mais changent les méthodologies et les outils d'interprétation pour montrer, cette fois-ci, non seulement l'objet mais l'histoire elle-même, la pratique, la mémoire, l'identité qu'il porte. Quand on parle d'un écomusée, on met toujours en cause une vision sacralisée du musée, un temple de la culture réservé à une élite. Il s'agit plutôt d'un lieu de démocratisation de la culture, utile à tous les questionnements de la société et à but socioculturel, scientifique et pédagogique. Au-delà d'être simplement une mode, les écomusées ont apporté des grands progrès à la muséologie. Ils prennent en compte l'espace et le temps en relation avec un territoire quelconque, en contribuant à son développement, et l'identité de la population qui y habite, ainsi que la participation de cette population au processus de patrimonialisation. Il s'agit aussi d'une démarche interdisciplinaire qui utilise les ressources de toutes les disciplines pour déchiffrer la réalité, pour la montrer au peuple de tous les côtés et dans tous les sens.

2 Cette idéologie, née en France, s'est répandue à l'international et continue à se diffuser en dehors du contexte francophone, qui a longtemps été le domaine privilégié de son utilisation. 


\begin{abstract}
« [...] And equally meaningful was the museological revolution started by the ecomuseums, with the opening of the Ecomusée de la Communauté Le Creusot Montceau in France in 1972 in an area rich in industrial remains. Ironbridge and Le Creusot Montceau represent two different approaches to similar problems, namely conceiving of a museum embracing a 'collection' made up of open-air structures preserved and interpreted in situ, a large area of land with its combination of natural and industrial landscapes, and even an entire community with its way of life, its memories, its contradictions ${ }^{2}$.»
\end{abstract}

3 Massimo Negri, qui s'exprime ici, explique ce qu'ont été les prémices de l'apparition des écomusées. Il part de la crise de la représentation du patrimoine industriel dans des musées traditionnels, causée par une approche interdisciplinaire de l'étude des objets. Un autre problème important pour les musées traditionnels résidait dans l'exposition des objets de grande taille. Quand on parle du patrimoine industriel, les spécialistes se heurtaient souvent à ce défi.

4 Comment créer une exposition muséale d'un chemin de fer, d'un moteur ou d'une rangée de maisons de la classe ouvrière ? Comment transmettre l'atmosphère et le savoir-faire à l'intérieur d'une usine, d'une fabrique, d'un atelier ?

5 Toutes ces questions ont trouvé leurs réponses dans cette nouvelle approche des musées qui a correspondu à la révolution des écomusées. Georges-Henri Rivière, père fondateur du musée des arts et traditions populaires, à Paris, et acteur principal dans le développement du concept d'écomusée, en donne la définition suivante :

\begin{abstract}
«Un écomusée est un instrument qu'un pouvoir et une population conçoivent, fabriquent et exploitent ensemble. (...) Un miroir où cette population se regarde, pour s'y reconnaître, où elle recherche l'explication du territoire auquel elle est attachée, jointe à celle des populations qui l'ont précédée, dans la discontinuité ou la continuité des générations. Un miroir que cette population tend à ses hôtes, pour s'en faire mieux comprendre, dans le respect de son travail, de ses comportements, de son intimité3. »
\end{abstract}

6 Proposée par Georges-Henri Rivière, cette définition de l'écomusée tenait compte des divers réflexions et apports collectifs, résultats des colloques sur l'écomusée organisés au Creusot de novembre 1975 à janvier 1976. Il insiste aussi sur le fait que l'écomusée renvoie à une expression de l'homme et de la nature, une expression du temps, une interprétation de l'espace, un conservatoire pour le patrimoine et une école qui implique la population dans les actions d'étude et de protection.

\title{
L'évolution des écomusées dans le monde
}

7 L'écomusée est donc né en France, en 1971, lors de la Conférence générale de l'Icom, d'où vient l'idée de revendiquer un rôle important pour les musées de sciences naturelles dans l'éducation à l'environnement, à l'écologie. Ce mouvement s'est manifesté d'abord dans des pays comme la France, la Norvège et la Suède, le Portugal, l'Italie et l'Espagne. À partir des années 1980, les écomusées se sont installés en Amérique, au Canada et en Brésil. Ce n'est qu'à partir depuis les années 1990 que le mouvement gagne les autres pays de l'Europe, y compris l'Europe orientale, ainsi que 
l'Inde et le Japon -chacun de ces pays essayant de donner sa propre adaptation et ses concepts à l'égard des écomusées.

8 L'Europe est certainement le continent le plus conservateur, surtout l'Europe occidentale, alors que l'Amérique Latine apparaît comme le continent le plus innovant, où s'est installée la majorité des écomusées urbains. L'Asie et le Pacifique commencent à expérimenter en fonction de leurs problèmes propres et de leurs cultures. Les pays anglo-saxons sont les plus réticents, les pays latins les plus enthousiastes. L'Europe orientale, restée longtemps à l'écart du mouvement de la nouvelle muséologie, l'a rejoint peu à peu. On y observe un développement des écomusées ruraux, point qui sera ensuite abordé. L'Afrique, à l'exception du Sénégal, est absente, peut-être parce que ses musées sont très pauvres en moyens et en personnels et sont encore proches de l'état où ils étaient à l'époque coloniale. Ces pays sont très liés de cette identité mixte davantage qu'à leur propre culture.

\section{Les écomusées en Europe de l'Est}

9 Dans les années 1960, le nombre des musées en plein air est en croissance dans certains pays d'Europe, notamment en Roumanie, où ce type de musées ruraux apparaissait en tant que souci de recherche scientifique sur les sociétés rurales et préindustrielles. Comme mentionné plus haut, les écomusées, en Europe orientale, sont apparus dans les années 1990, avec une implantation plutôt en milieu rural.

10 En Russie, ce type de musées est apparu en tant que nouvelle méthodologie de base pour le développement des musées en plein air et des musées-réserve. Le concept de musées en plein air remonte au $\mathrm{XV}^{\mathrm{e}}$ siècle et part des musées-réserve (russe: ЗАПОВЕДНИК), ces derniers étant utilisés uniquement en faveur des objets de la nature, signifiant les grandes surfaces de forêts qu'il était interdit de défricher. Au cours de la seconde moitié du XXe siècle, la forme d'organisation des musées-réserve était optimale pour l'organisation de la protection, du développement, et l'utilisation muséale des territoires naturels et culturels ainsi que des éléments immobiliers de grande valeur scientifique, esthétique voire commémorative. Parmi ceux-ci apparaissent les sites archéologiques des anciennes colonies; les lieux les plus connus des batailles; les musées-palais ou manoir; les ensembles monastiques; les paysages naturels et artificiels importants, etc.

11 À la fin du XXe siècle a eu lieu l'implémentation des processus de transformation des musées-réserve dans des musées "vivants et intégrés ", en relation avec les pratiques modernes des musées. L'écomuséologie est apparue ensuite comme une nouvelle direction de la muséologie ainsi que la base théorique et méthodologique des changements dans le bâtiment des musées. Les écomusées sont devenus les centres nationaux et culturels pour la conservation, l'étude et l'utilisation des objets historiques dans leur environnement culturel et naturel d'existence. Par exemple, on peut citer les écomusées situés dans la Russie européenne : Écomusées de Novgorod, Soudzal (Moscou) et de l'ile de Kiji.

\section{L'Écomusée de l'île de Kiji}

12 Au nord de Saint-Pétersbourg, près de la Finlande, la Carélie est une terre marécageuse et boisée. Plus d'un sixième de son territoire est couvert de lacs. La petite île de Kiji est 
longue de 5,5 km environ et sa largeur n'excède pas 1,4 km. Elle se trouve au nord-est de l'immense lac Onéga, le deuxième d'Europe après le lac Ladoga.

13 Cette réserve historique est un des premiers musées à ciel ouvert de Russie. L'écomusée abrite deux églises en bois du XVIII siècle et un clocher octogonal, également en bois, assemblé en 1862.

\section{Fig.1. Carte de l'écomusée de Kiji}

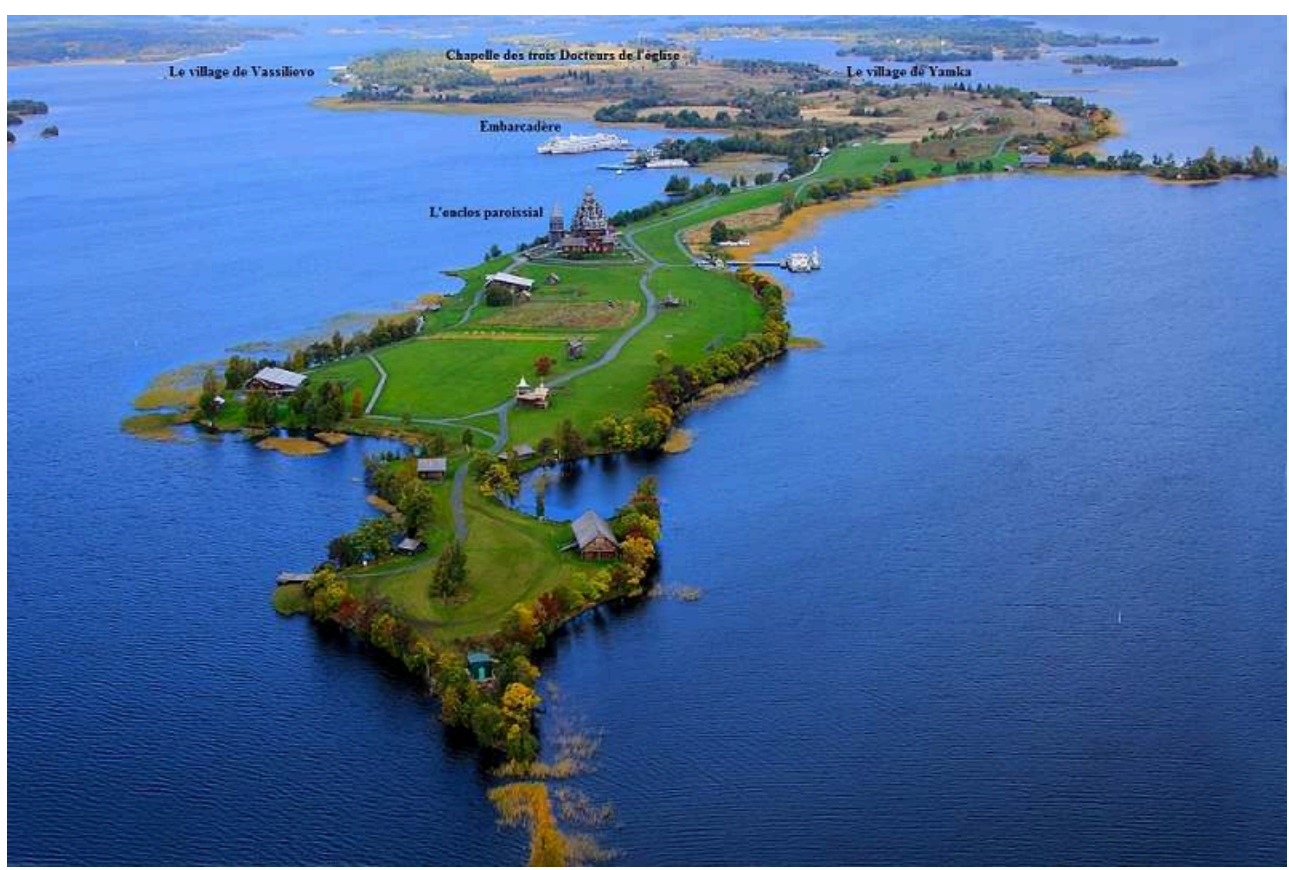

La réserve historique Kiji est un des premiers musées à ciel ouvert de Russie. Le musée a plus de 1500 mètres carrés comme espace d'exposition sur lîle de Kiji et sur les petites îles voisines. Actuellement, l'écomusée abrite deux églises en bois du XVIIIe siècle et un clocher octogonal, également en bois, assemblé en 1862. II comprend des œuvres d'architecture en bois de toute la Carélie et se compose de plusieurs secteurs d'exposition, y compris deux villages d'exposition, Vassilievo et Yamka, et de monuments distincts des Vepses, de la Carélie du Nord et du folklore carélien. Au total, il y a 87 monuments d'architecture populaire en bois sur le territoire du musée, plus de 500 icônes uniques et anciennes, et une exposition vivante qui raconte la vie, les traditions et la culture de cette région.

(c) L'auteur, 2018

Le musée a été fondé le $1^{\text {er }}$ Janvier 1966 sous le nom d'«Ensemble Historicoarchitectural et ethnographique du musée Kiji » (en russe: госУдАРстВЕНнЫй ИСТОРИКО-АРХИТЕКТУРНЫЙ И ЭТНОГРАФИЧЕСКИЙ МУЗЕЙ-ЗАПОВЕДНИК «КИЖИ»). L'ile de Kiji est devenue célèbre pour son musée en plein air classé depuis 1990 au patrimoine mondial de l'UNESCO.

Le joyau de cet ensemble unique est l'église de la Transfiguration, ouvrage de charpente coiffé de 22 bulbes argentés formant une sorte de pyramide. Elle contient une belle iconostase baroque. L'église de l'Intercession de la Vierge, surmontée de neuf bulbes, a été édifiée à proximité en 1764. La couverture de ces deux édifices est ellemême en bois, et le tout a été assemblé sans clous, ni vis ou pièce métallique. 
Fig 2. L'église de la Transfiguration (1714)

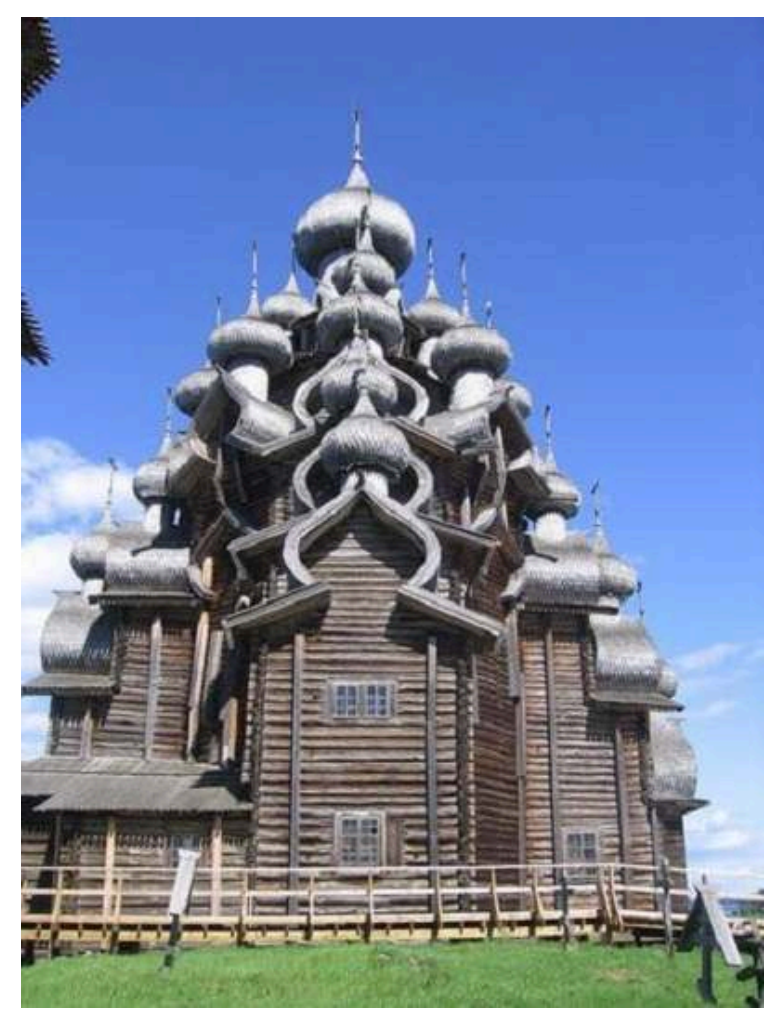

L'ensemble des églises avec ses cimetières (cet ensemble est généralement nommé Pogoste depuis le Xle siècle), occupe une place centrale dans Kiji. Le monument principal du Pogoste est l'église de la Transfiguration. Elle a été construite en pin, en 1714, à la place d'une église plus ancienne brûlée à la suite d'un impact de foudre. Sa hauteur est de $37 \mathrm{~m}$. Les dômes de l'église sont placés sur 5 niveaux et chaque dôme de chaque niveau a une taille différente. Le premier et le deuxième niveau comprennent quatre dômes ; le troisième, huit ; le quatrième, quatre ; le cinquième, un seul. Le dernier dôme est situé au-dessus de l'autel de l'église. Â l'intérieur de l'église, il y a un plafond pyramidal à multiples facettes avec des vestiges de peintures murales, appelés « ciel ». Le plafond augmente l'espace vertical de l'église. L'iconostase de l'église à quatre niveaux date du milieu du XVIIII siècle. II est composé de 102 icônes. Les deux icônes les plus anciennes - «Transfiguration » et « Couverture »appartiennent à la fin du XVII ${ }^{\mathrm{e}}$ siècle.

(C) Lizotchka, 2008

Cet écomusée propose des expositions, conférences et animations pour faire vivre le lieu et pour impliquer la population. Voici quelques exemples :

- Le programme "retour aux sources » (en russe: ПРОГРАММА « ВОЗВРАЩЕНИЕ К истокАм ») propose des discussions avec les habitants et permet une sensibilisation des jeunes à l'environnement. Tout cela contribue à l'interactivité du musée avec les autres républiques de l'ancienne URSS. Le but est de faire prendre conscience à la population de l'intérêt de perpétuer les traditions.

- L'écomusée sur le lac Onega possède une succursale à Petrozavodsk. Il propose l'exposition «Un jour dans le quotidien d'une famille du lac Onega à la fin du XIX ${ }^{e}$ siècle/début du XX" $"$, etc.

Kiji (ou "Kizhi» selon la translittération officielle) est connue pour son musée qui rassemble des exemples de l'architecture typique de la Russie du Nord. Outre ses collections exceptionnelles, la position géographique de l'île, tout au Nord, lui confère une lumière toute particulière qui contribue à l'enchantement du spectateur.

En Ukraine, les écomusées n'ont pas encore reçu de reconnaissance. Il y a des formes de conservation $\mathrm{du}$ patrimoine paysager qui sont typologiquement proches de 
l'organisation des écomusées. Elles existent grâce à l'organisation des parcs paysagers régionaux. Par contre, en Ukraine, les musées au profil littéraire, musical, orientés vers les sciences naturelles sont très répandus.

Comme exemple de musée en plein air, le musée de l'architecture populaire et de la vie quotidienne Pirogov (Pirogovo) situé à Kiev, en Ukraine, rappelle l'organisation des écomusées. Fondé en 1969, il s'étend sur 150 hectares. C'est l'un des plus grands musées en plein air au monde. Le musée recueille une immense collection de vêtements nationaux, de meubles, de vaisselle et d'instruments de musique. Ce musée possède plusieurs des grands principes des écomusées : il implique la population et raconte l'histoire du territoire via des représentations théâtrales qui sont proposées aux visiteurs mettant en valeur des traditions anciennes. Lors des fêtes, on y entend des chansons nationales. Forgerons, potiers, fondeurs, tisseurs et autres maîtres présentent leurs savoir-faire au public en tant que grands métiers du passé de l'Ukraine. Tous les dimanches, des concerts collectifs de folklore ukrainien sont organisés. Les visiteurs du musée peuvent aussi déguster les plats de la cuisine traditionnelle ukrainienne dans les restaurants situés sur le territoire du musée, à moins qu'ils ne préfèrent pique-niquer en plein air. Un show équestre et la visite de l'atelier d'un forgeron sont également proposés. Le territoire du musée Pirogov accueille une exposition unique de moulins à vent.

On y retrouve aussi une vieille maison russe en bois du XI ${ }^{e}$ siècle, la maison de la fin du $\mathrm{XVI}^{\mathrm{e}}$ siècle construite pour une personne, une église en bois de la région de Kiev datée de l'an 1600 et plusieurs églises du XVIII siècle transportées des Carpates, de Polésie, de la région de Tcherkassy: l'église Archange Saint-Michel, l'église Saint-Nicolas, l'église Sainte martyre Parascève et l'église de l'Intercession de la Sainte Vierge.

21 La République de Moldavie reste le pays le moins concerné par " la fièvre " de l'écomuséologie. Elle dispose pourtant de nombreuses ressources pour en créer. Un exemple caractéristique se trouve dans le nord du pays, à côté de la ville d'Orhei. Il s'agit d'un musée en plein air, le plus célèbre du pays. Il possède une importance historique particulière.

"Orheiul Vechi » (l'Ancien Orhei) est un site archéologique situé à proximité du village de Trebujeni, à environ 60 kilomètres de Chişinău. Ce site conserve des vestiges des civilisations datant des époques les plus reculées qui reflètent l'histoire lointaine du territoire. On y a découvert des outils en pierre témoignant d'une civilisation très ancienne. Les visiteurs peuvent accéder aux ruines d'un village médiéval, y compris aux vestiges d'une mosquée, de deux mausolées, d'un caravansérail, de deux hammams et d'une maison moldave typique. Le site est aussi fameux grâce à une ancienne forteresse, bâtie par ştefan cel Mare au XIV ${ }^{\mathrm{e}}$ siècle, puis détruite pendant le siècle suivant par les Tatares, ainsi qu'à l'ermitage de Butuceni et un monastère rupestre du $\mathrm{XIII}^{\mathrm{e}}$ siècle ${ }^{4}$. Orheiul-Vechi est un monument naturel, historique et archéologique protégé par l'État. Ce site implanté au bord de la rivière le Răut a connu plusieurs cultures archéologiques. Différents peuples migrateurs s'y succédèrent: Sarmates, Gothes, Huns, Avars, Slaves, Hongrois, etc. 


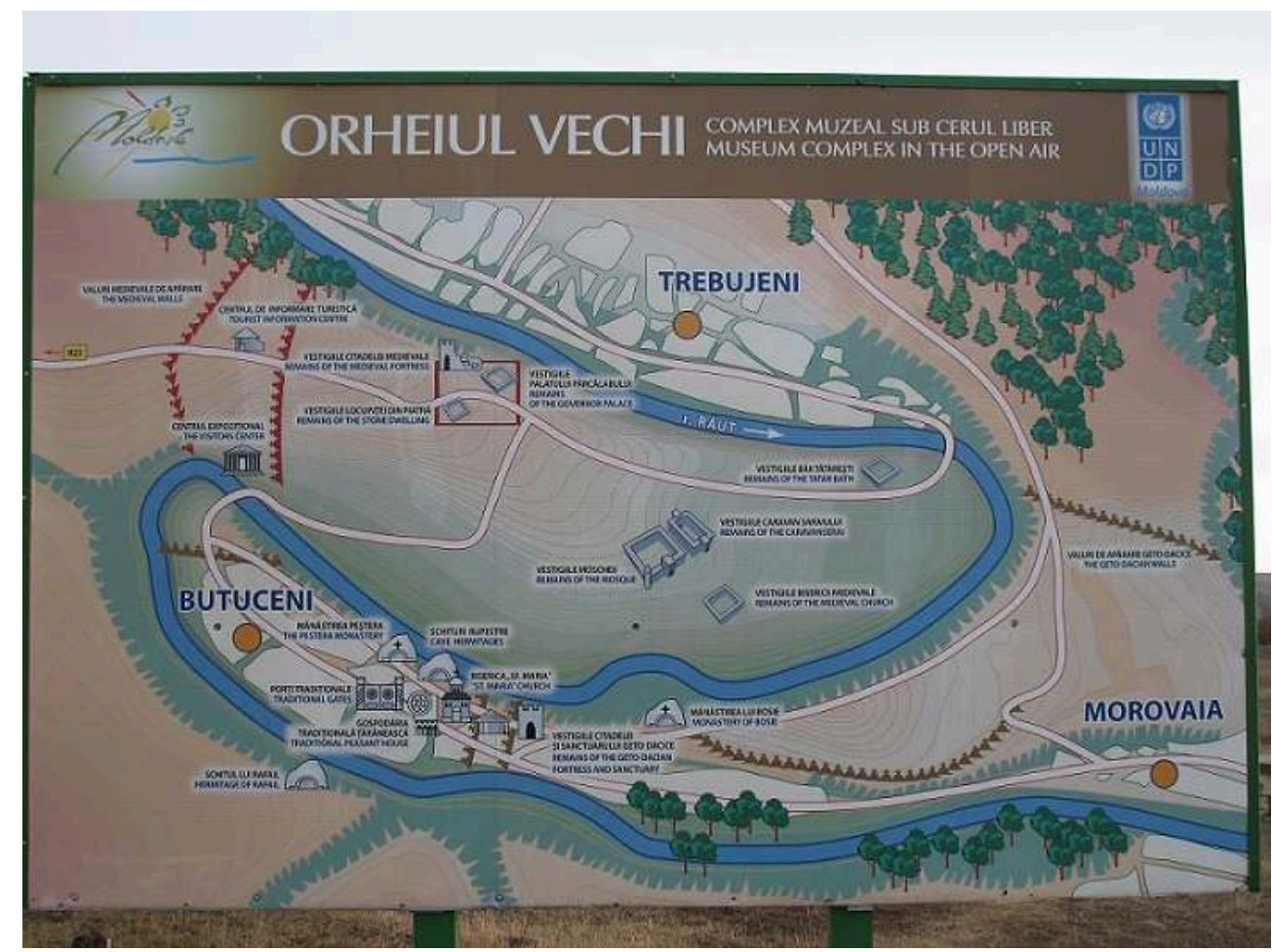

Le complexe de l'Ancien Orhei se compose de plusieurs dizaines d'hectares de la ville médiévale d'Orhei (XIIle-XVle siècles), plus tard baptisée Orheiul Vechi. Le complexe comprend deux grands promontoires (Cave et Butuceni), auxquels se joignent trois promontoires plus petits adjacents (Potarca, Selitra et Scoc), sur le territoire desquels se trouvent les ruines de fortifications, habitations, bains, lieux de culte, y compris des grottes), autant de la période tatare-mongole (XIII-XIVe siècles) et de celle moldave (XVe-XVle siècles).

(c) Domaine public, 2007

On peut voir, alors, que ce musée en plein air a tous les atouts pour devenir un écomusée moldave. Il contient déjà la majorité des éléments de base qui constituent un écomusée. Il répond aussi aux trois principes fondamentaux élaborés par Hugues de Varine pour un écomusée (fig.4). 
Fig.4. Les principes des écomusées d'après Hugues de Varine

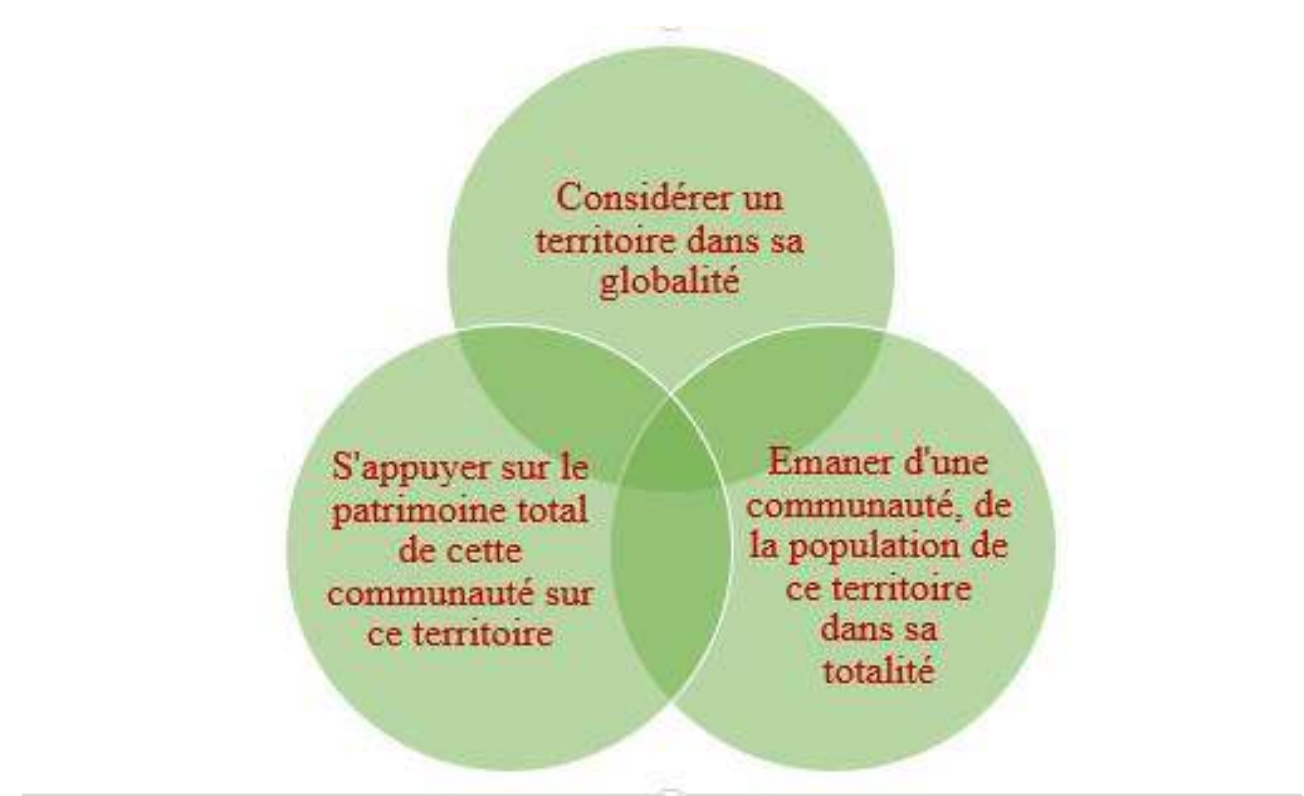

Schéma élaboré par l'auteur, 2016. République de Moldavie, sont les partenaires d'une initiative qui contribue à la création des écomusées, nommée greenway (la voie verte). Les greenways, fondées aux États-Unis dans les années 1950, sont liés à des initiatives des écomusées, qui visent à protéger et interpréter le patrimoine local à travers la participation des communautés locales. Leur but est d'améliorer la qualité de vie et de promouvoir le développement durable, l'écotourisme, la conservation du patrimoine naturel et culturel, ainsi que le soutien des communautés dans leur développement économique et social, y compris le 
développement des entreprises. Les écomusées sont utilisés pour donner de la valeur aux greenways régionaux, nationaux et internationaux.

D'après les spécialistes, pour les pays de l'Europe de l'Est, les greenways, connus dans le monde slave comme ЗЕЛЕНЫЕ МАРШРУТКИ, représentent le mode de réalisation de l'idée d'écomusée. C'est une plateforme qui réunit les intérêts d'un grand nombre de personnes; grâce aux « voies vertes » les petits villages et villes est-européennes sont en cours de développement, parce que cette plateforme laisse dans les régions $12 \%$ de ses revenus pour soutenir les petites communautés.

Fig. 5. Les principes des greenways
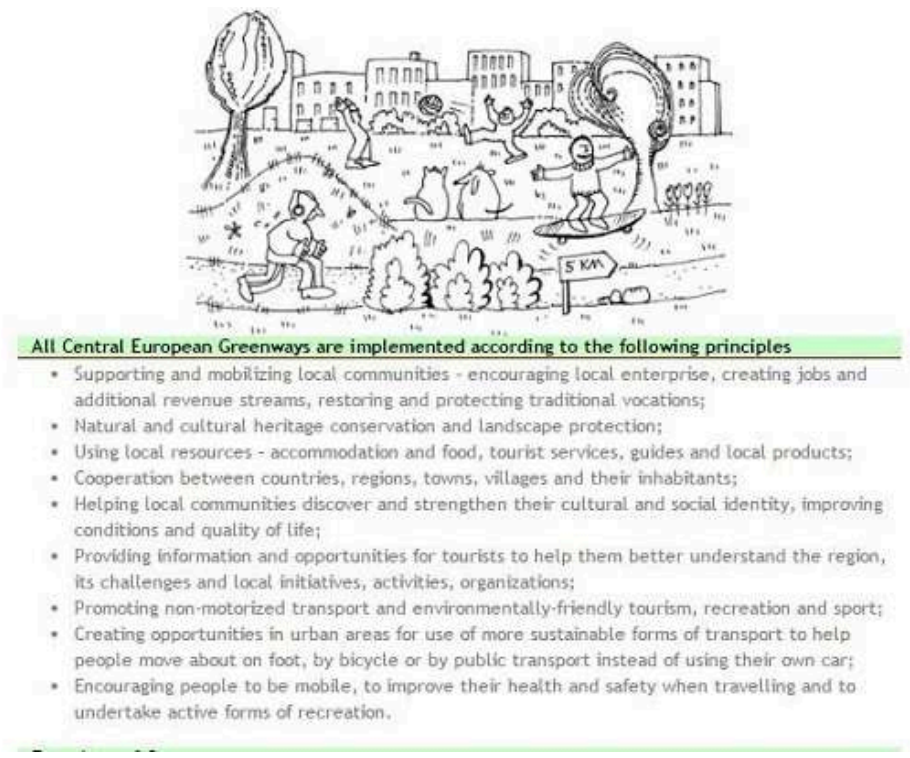

(c) greenways.by, 2016

Le long des greenways se développent des infrastructures; des cafés, musées, écomusées, hôtels sont ouverts. Grâce à l'afflux de touristes qui voyagent sur cette voie, des nouveaux emplois sont créés dans la région et on observe une croissance dans l'esprit entrepreneurial parmi les résidents ruraux. Le travail, dans l'avenir, poussé dans cette direction permettra aux pays comme l'Ukraine, la Biélorussie et la République de Moldavie de développer le concept et de créer des écomusées.

\section{BIBLIOGRAPHIE}

BILLARD Alexandra, «Kiji, des origines à nos jours», article posté sur le site voyageur.com, mis en ligne le 11/05/12, http://www.e-voyageur.com/magazine-voyage/russie/kiji-musee-ciel-ouvertcarelie-3, consulté le 25 juin 2016

CHAUMIER Serge, Une nouvelle approche de l'écomusée, des musées en quête d'identité : écomusée versus technomusée, Paris, Ed. L'Harmatan (Nouvelles études anthropologiques), 2003 
DELARGE Alexandre, « Des écomusées, retour à la définition et évolution », p.139-155, Publics et Musées, n¹7-18, 2000, p.139-155

NEGRI Massimo, « Industrial Heritage Retooled », TICCIH Bulletin, $\mathrm{n}^{\circ} 70$ - 4th quarter, 2015, Michigan

POSTICA Gheorghe, Orheiul Vechi. Cercetări arheologice (1996-2001). Iași: Editura UniversităȚii A.I. Cuza, 2006, [En français : POSTICA Gheorghe, L'Ancien Orhei. Recherches archéologiques (1996-2001), Iasi, Ed. Université A.I. Cuza, 2006.]

VARINE Hugues de, «L'Écomusée. Un mot, deux concepts, mille pratiques », Mus-A, Revista de los museos de Andalucia, Sevilla, ${ }^{\circ} 8,2007$, p.19-29. (Texte d'une intervention à une rencontre des musées d'Andalousie à Grenade)

АлмАТА, 2012, nr. 125, p.6. (En français : Vestnik CazNU, Almaty)

БАЗАРБЕККЫЗЫ, А., «ВОПЛОЩЕНИЕ ИДЕИ ЭКОМУЗЕЕВ В ЕВРОПЕ (НА ПРИМЕРЕ СЕТИ «ЗЕЛЕНЫХ МАРШРУТОВ»)» [En français : Bazarkbekyzy, A., «Incarnation de l'idée des écomusées en Europe (en utilisant l'exemple d'un réseau de "routes vertes") »], publié dans le journal ВЕСтник КАЗну

КАУЛЕН, Г.Р. РУДЕНКО, И.В. ЧУВИЛОВА, МУЗЕИ-ЗАПОВЕДНИКИ - МУЗЕИ БУДУЩЕГО:

МЕЖДУНАРОДНАЯ НАУЧНО-ПРАКТИЧЕСКАЯ КОНФЕРЕНЦИЯ. ЕЛАБУГА, 18-22 НОЯБ. 2014 Г.:

МАТЕРИАЛЫ И ДОКЛАДЫ / ОТВ. РЕД. М.Е.. - ЕЛАБУГА: ООО «ЕЛТИК», 2015. - 324 С. [Еn français :

Kaulen, G.R., Rudenko, I.V., Chuvilova, Les musées réserve-les musées de l'avenir : Conférence internationale scientifique et pratique. Yelabuga, 18-22 nov. 2014, matériau publié en 2015.]

КУКЛИНОВА И. А., «ЭКОМУЗЕЙ ВРЕМЕНИ И ПРОСТРАНСТВА: ЗАПАДНОЕВРОПЕЙСКИЙ ОБРАЗЕЦ И ВОСТОЧНАЯ МОДЕЛЬ», ТРУДЫ САНКТ-ПЕТЕРБУРГСКОГО ГОСУДАРСТВЕННОГО УНИВЕРСИТЕТА культУРы и искусстВ, том 190 / 2010, p.263-265. (En français : KUKLINOVA I.A., «Écomusée dans l'espace et le temps : modèle de l'Europe occidentale et orientale », Actes de l'Université d'État de la culture et des arts de Saint-Pétersbourg, vol. 190/2010, p.263-265.)

\section{NOTES}

1. VARINE Hugues de, «L'Écomusée. Un mot, deux concepts, mille pratiques », Mus-A, Revista de los museos de Andalucia, Sevilla, n8, 2007, p.20.

2. NEGRI Massimo, «Industrial heritage retooled», TICCIH Bulletin, $\mathrm{n}^{\circ} 70-4^{\text {th }}$ quarter, 2015, Michigan, p.167.

3. DELARGE Alexandre, «Des écomusées, retour à la définition et évolution », Publics et Musées, $\mathrm{n}^{\circ} 17-18,2000, \mathrm{p} .139-155$.

4. Article publié dans le Portail de la Moldavie francophone, consultable sur le lien: http:// www.moldavie.fr/Orheiul-Vechi-un-musee-en-plein-air.html, (l'auteur et la date de la publication ne sont pas indiqués).

5. Voir le site officiel, disponible en anglais, russe, ukrainien et biélorusse: http:// www.greenways.by. 


\section{RÉSUMÉS}

Ce travail porte sur les écomusées en Europe de l'Est, partie du monde assez méconnue des occidentaux. Il part de l'histoire des écomusées avec l'apparition du concept en France, ainsi que la création du premier écomusée Le Creusot-Montceau, jusqu'à leur développement un peu partout dans le monde, pour s'arrêter enfin sur des exemples concrets en Europe orientale, notamment en Russie européenne, Ukraine, Biélorussie et République de Moldavie. Pour ces trois derniers pays l'écomusée et l'écomuséologie ne représentent pas encore "la nouvelle mode ", mais ils trouvent des moyens et des plateformes qui leur permettront, dans l'avenir, d'implémenter cette nouvelle approche unique et exceptionnelle. Il y existe, d'ailleurs, des exemples éminents qui méritent d'être mis en évidence.

This work is focused on the ecomuseums in Eastern Europe, a part of the world quite unknown for the occidentals. It starts with the history of ecomuseums since the apparition of the concept in France, and the creation of the first ecomuseum Le Creusot-Montceau; their development around the world, and it stops after, on concrete examples in Eastern Europe: the European part of Russia, Ukraine, Belarus and Moldova. For the last three countries in this list, the ecomuseums and the ecomuseology does not yet represent the "new fashion", but they are finding ways and platforms enable to help them to implement, in the future, this new, unique and exceptional approach. There are, however, outstanding examples that deserve to be highlighted.

\section{INDEX}

Mots-clés : histoire des techniques, écomusée, voie verte, patrimoine immatériel, musée de plein air, muséologie

Keywords : history of technology, eco-museum, intangible heritage, greenway, museology, openair museum

\section{AUTEUR}

\section{MIHAELA NICHIFOR}

Diplômée du Master Erasmus Mundus TPTI (Techniques, Patrimoines et Territoires de l'Industrie), promotion Palmyre, avec le mémoire Conservation et valorisation du patrimoine immatériel moldave : l'ensemble de danses folkloriques « Joc ». Actuellement employée à la Présidence de la République de Moldavie. 